\title{
Early surfactant replacement guided by lung ultrasound in preterm newborns with RDS: the ULTRASURF randomised controlled trial
}

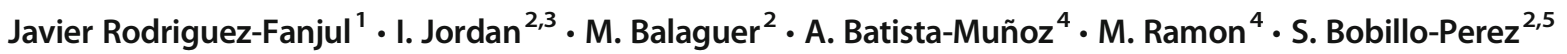

Received: 1 April 2020 / Revised: 13 July 2020 / Accepted: 16 July 2020 / Published online: 24 July 2020

(C) Springer-Verlag GmbH Germany, part of Springer Nature 2020

\begin{abstract}
This study aimed to investigate whether using lung ultrasound (LUS) scores in premature newborns with respiratory distress syndrome (RDS) allows for earlier surfactant therapy (within the first $3 \mathrm{~h}$ of life) than using $\mathrm{FiO}_{2}$ criteria. This was a randomised, non-blinded clinical trial conducted in a neonatal intensive care unit. The inclusion criteria were newborns with a gestational age of $\leq 32$ weeks and RDS. Patients meeting the inclusion criteria were randomly assigned to two groups: the ultrasound group, administered surfactant based on LUS score and/or $\mathrm{FiO}_{2}$ threshold, and the control group, guided by $\mathrm{FiO}_{2}$ only. Fifty-six patients were included. The ultrasound group received surfactant earlier ( $1 \mathrm{~h}$ of life vs. $6 \mathrm{~h}, p<0.001)$, with lower $\mathrm{FiO}_{2}(25 \%$ vs. $30 \%, p=$ $0.016)$ and lower $\mathrm{CO}_{2}$ (48 vs. $\left.54, p=0.011\right)$. After surfactant treatment, newborns in the ultrasound group presented a greater $\mathrm{SpO}_{2}(p=0.001)$ and $\mathrm{SpO}_{2} / \mathrm{FiO}_{2}$ ratio $(p=0.012)$.

Conclusions: LUS score allowed an earlier surfactant therapy, reduced oxygen exposure early in life and a better oxygenation after the treatment. This early surfactant replacement may lead to reduced oxygen exposure.
\end{abstract}

\section{What is Known:}

- Lung ultrasound scores predict the need for surfactant therapy in premature newborns.

What is New:

- This study shows that using lung ultrasound scores improves the timeliness of surfactant replacement compared with using $\mathrm{FiO}_{2}$ alone.

Keywords Lung ultrasound $\cdot$ Respiratory distress syndrome $\cdot$ Premature newborns $\cdot$ Surfactant

\author{
Abbreviations \\ CXR Chest X-ray \\ GA Gestational age
}

LUS Lung ultrasound

NA Neonatologist-assistant

nCPAP Nasal continuous positive airway pressure

\footnotetext{
Communicated by Daniele De Luca

Javier Rodriguez-Fanjul

javier.rodriguez.fanjul@gmail.com

I. Jordan

ijordan@sjdhospitalbarcelona.org

M. Balaguer

mbalaguer@sjdhospitalbarcelona.org

A. Batista-Muñoz

albertbatis.hj23.ics@gencat.cat

M. Ramon

mramonjimenez.hj23.ics@ gencat.cat

S. Bobillo-Perez

sbobillo@fsjd.org
}

1 Neonatal Intensive Care Unit, Paediatrics Department, Hospital Germans Trias i Pujol, Universitat Autònoma de Barcelona, Badalona, Spain

2 Paediatric Intensive Care Unit, Hospital Sant Joan de Déu, Universitat de Barcelona, Barcelona, Spain

3 Paediatric Infectious Diseases Research Group, Institut de Recerca Hospital Sant Joan de Déu, CIBERESP, Barcelona, Spain

4 Neonatal Intensive Care Unit, Paediatrics Department, Hospital Joan XXIII, Tarragona, Spain

5 Disorders of Immunity and Respiration of the Paediatric Critical Patient Research Group, Institut de Recerca Hospital Sant Joan de Déu, Universitat de Barcelona, Barcelona, Spain 
NICU Neonatal intensive care unit

NR Neonatologist-researcher

RDS Respiratory distress syndrome

\section{Introduction}

The management of respiratory distress syndrome (RDS) in premature newborns is centred on the use of nasal continuous positive airway pressure (nCPAP) [1]. Determining the need for surfactant treatment is currently guided only by the newborn's requirement for oxygen therapy. In recent guidelines, early therapy with surfactant is suggested in patients requiring nCPAP of at least $6 \mathrm{cmH}_{2} \mathrm{O}$ and an $\mathrm{FiO}_{2}$ over 0.3 [2]. Certainly, this cut-off can be considered arbitrary, given that the current evidence is based on observational studies that considered $\mathrm{FiO}_{2}$ requirements greater than 0.3 as a predictor of respiratory failure [3] and may not accurately reflect the patient's oxygenation [4]. Therefore, it is challenging for neonatologists to identify the newborns who will benefit from early surfactant treatment (within the first $3 \mathrm{~h}$ of life), which is known to decrease pneumothorax and bronchopulmonary dysplasia and to improve survival rates [3]. However, patients receiving surfactant may present side effects including bradycardia, oxygen desaturation and pulmonary haemorrhage, among others [5].

For decades, clinicians have used chest X-rays (CXR) to identify RDS, as they show the pathology's characteristic diffuse reticulogranular pattern or ground glass appearance. However, this technique has low sensitivity and specificity [6]. Moreover, thanks to improvements in standard prenatal and postnatal care, the RDS-like CXR image has also become more uncommon [7].

Lung ultrasound (LUS) has emerged as a bedside technique that helps clinicians to identify RDS patients in neonatal intensive care units (NICUs) [8-12]. Some studies have shown a high correlation between LUS findings and oxygenation status/need for surfactant therapy $[13,14]$. Furthermore, some studies have shown LUS score to be a more accurate diagnostic criterion than $\mathrm{FiO}_{2}$ [10, 13-15], and the latest European respiratory distress guidelines highlight its potential use in identifying patients with RDS [2]. A recent high-quality prospective study showed that LUS improved timeliness in surfactant administration in a tertiary NICU during two time periods [16], but no clinical trial has evaluated the use of surfactant therapy guided by LUS only versus conventional $\mathrm{FiO}_{2}$ guidance.

Moreover, there is an increased understanding about the potential lifelong impact of excessive oxygen exposure during the first hours of life in preterm infants, and LUS may help to avoid this oxygen toxicity [17] by reducing exposure to it.
This study aims to investigate if using LUS score ensures timely surfactant administration in premature patients better than $\mathrm{FiO}_{2}$ does.

\section{Materials and methods}

\section{Design}

This was a randomised, non-blinded clinical trial conducted in the NICU of Hospital Joan XXIII, Tarragona, in Spain, between January 2019 and March 2020. The study was carried out in accordance with the Declaration of Helsinki and was approved by the local ethics committee and institutional review board (EIM 136/2019). The study was registered at ClinicalTrials.gov, with identifier NCT04330443. Written informed consent was obtained prenatally from the patients' parents or guardians.

\section{Patients}

Inclusion criteria were premature newborns under the gestational age (GA) of 32 weeks with RDS [18], defined as respiratory distress appearing within the first $24 \mathrm{~h}$ of life which required nCPAP to keep peripheral oxygen saturation $\left(\mathrm{SpO}_{2}\right)$ above $90 \%$, with clinical signs of respiratory difficulty (polypnoea, chest retractions, nasal flutter, etc.) and a complete, sustained and prompt response to surfactant and/or lung recruitment. An additional, non-mandatory criterion was lung images that support the diagnosis.

Exclusion criteria were as follows: non-acceptance of the informed consent, chromosomal abnormalities, complex congenital malformations, signs of early-onset septic shock, mechanical ventilation and patients who received surfactant in the delivery room as part of advanced resuscitation following the pertinent European guidelines [2].

\section{Objectives}

The main objective of the study was to determine if a diagnosis of RDS guided by a LUS algorithm allowed for earlier surfactant therapy (within the first $3 \mathrm{~h}$ of life) in comparison with using an $\mathrm{FiO}_{2}$ threshold alone.

The secondary objective was to assess the different levels of oxygen exposure in early life for each group. Other secondary outcomes were $\mathrm{SpO}_{2} / \mathrm{FiO}_{2}$ ratio $(\mathrm{S} / \mathrm{F}$ ratio) after surfactant therapy (see below), the need for mechanical ventilation (MV) (defined as MV during the first 3 days of life [18]), duration of invasive and non-invasive mechanical ventilation, ventilator-free days [19], duration of supplemental oxygen requirements, length of stay in the NICU and bronchopulmonary dysplasia [20]. 


\section{Methodology}

Patients who met the inclusion criteria were randomly assigned to two groups, using the "random" function in the MS-Excel ${ }^{\circledR}$ programme. A binary sequence of random numbers was generated following a balanced block sampling (Friedman procedure) and held by the principal investigator. A total of 6 physicians enrolled participants, all them fully trained in the use of LUS. The principal investigator assigned participants to interventions based on the randomised list. Patients with a gestational age of under 32 weeks were included as they were born during the study period:

- Ultrasound group: The neonatologist-researcher (NR) who was not the attending clinician performed the LUS at admission during the first hour of life. The neonatologistassistant (NA) for the baby was not blinded to the result of the LUS. Patients received surfactant therapy as soon as the LUS score was higher than 8 or $\mathrm{FiO}_{2}$ requirements exceeded $30 \%$ in patients with a GA of $<28^{6 / 7}$ weeks or $40 \%$ in patients whose GA was $>29^{0 / 7}$ weeks (Fig. 1) [22]. This LUS threshold has been found to be the one with the best diagnostic accuracy $[10,13,14]$.

- Control group: The NR performed the LUS at admission during the first hour of life. The NA was not blinded to the result of the LUS. The patient received surfactant therapy only when $\mathrm{FiO}_{2}$ requirements exceeded 30\% in patients with a GA of $<28^{6 / 7}$ weeks or $40 \%$ in patients whose GA was $>29^{0 / 7}$ weeks (Fig. 1).

\section{General patient management}

Our unit follows the European guidelines for respiratory support in premature newborns [2]. In the delivery room, patients received face mask ventilation with continuous positive airway pressure and a target peak inspiratory pressure.

Unresponsive patients were intubated in the delivery room and received early surfactant administration, so they were excluded from the study. Those who were responsive at birth were transferred to the NICU on nCPAP if they were born before 32 weeks of GA.

Once admitted to the unit, patients were placed under variable flow nCPAP using the appropriate nasal prongs or facial mask with a pressure between 5 and $7 \mathrm{cmH} 20$, with oxygen being increased as needed to keep $\mathrm{SpO} 2$ between 90 and $95 \%$.

Patients were intubated if $\mathrm{FiO}_{2}>50 \%$ despite surfactant therapy, if they had multiple episodes of apnoea ( $>4$ episodes per hour or $>1$ episode requiring rescue with positive-pressure ventilation) or if respiratory acidosis was detected ( $\mathrm{pCO} 2>$ $65 \mathrm{mmHg}$ and $\mathrm{pH}<7.20$ in capillary samples).

The LUS study protocol included longitudinal scans of the anterior, lateral and posterior chest walls performed with a lineal $12 \mathrm{MHz}$ probe (Sonosite Edge II) during the first hour

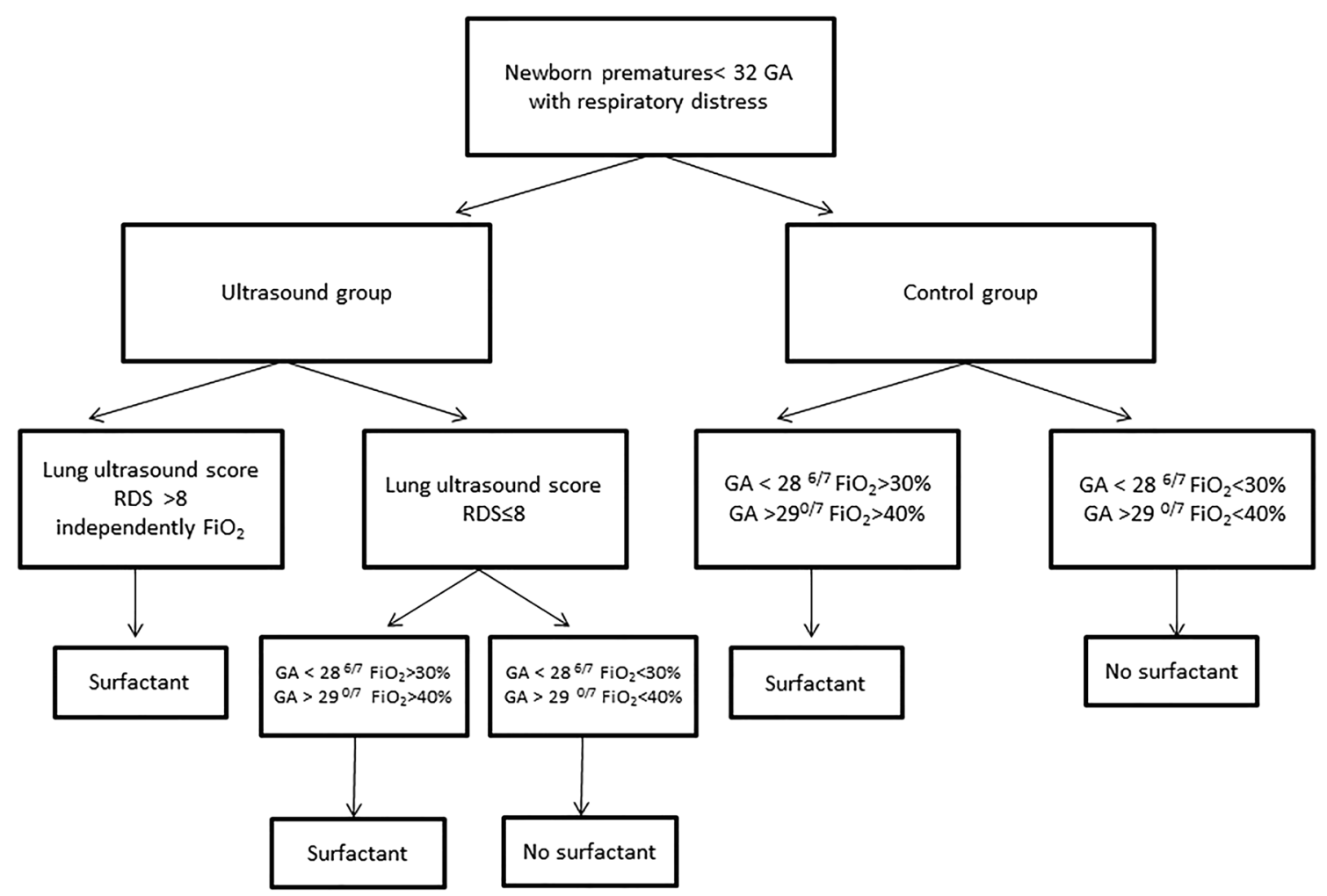

Fig. 1. Study protocol algorithm. RDS, respiratory distress syndrome; GA, gestational age 
of life once patients were in the NICU. Focus was placed on the pleural line, and no harmonics were used. Three scans were performed per hemithorax (anterior, lateral and posterior) and a 0 - to 3-point score was given for each scanned area (with a total score ranging from 0 to 18), in accordance with previous studies published in the literature $[11,13,14] ; 8$ was the cut-off score for initiating surfactant treatment [16].

Clinical patient data were recorded, including $\mathrm{SpO}_{2}$ and $\mathrm{S} / \mathrm{F}$ ratio [21] at the time of the LUS scan and when the patient received surfactant therapy, $5 \mathrm{~min}$ after the procedure. $\mathrm{CO}_{2}$ and $\mathrm{pH}$ were analysed before surfactant administration, using arterialised capillary blood samples. We acknowledge that S/F is an imperfect oxygenation metric, as it may be influenced by foetal haemoglobin, peripheral perfusion, patent ductus arteriosus, temperature and other factors. Surfactant therapy was exclusively administered through less invasive surfactant administration (LISA). The first dose given was $200 \mathrm{mg} / \mathrm{kg}$ of poractant alfa (Curosurf $®$, Chiesi Farmaceutici, Parma, Italy) [22]. Bradycardia was defined as a heart rate of less than $80 \mathrm{bpm}$ and desaturation was defined as a drop in oxygen saturation to $<85 \%$. These complications were considered to be related to the procedure if they were recorded during the next $30 \mathrm{~min}$ after the surfactant therapy. Ventilator-free days were calculated as the number of days in the NICU without invasive mechanical ventilation, within the first 28 days of life; this number is zero for patients who died in the NICU [19].

The sample size was calculated using the ene $3.0 ®$ programme. A sample size of 40 patients for each group was required if assuming $80 \%$ power to reject the null hypothesis (no differences between the lung ultrasound and control groups) and accepting an alternative hypothesis with a twotailed type I error (alpha) value as determined by the bibliography. We performed a pre-analysis to detect differences between data from previous studies [16] and our data, observing a relatively delayed surfactant administration in our control group (timely administration in 6\% vs. $71 \%$ of cases reported by the ESTHER study [16]). This exploratory analysis included the 56 patients in our study, 27 and 29 patients in each respective group, giving us a statistically significant sample size.

The statistical analyses were performed using the SPSS $®$ 26.0. Categorical variables were expressed as frequency (percentage) and compared using the chi-squared test or Fisher's exact test, as needed. Continuous variables were expressed as median (interquartile range) and compared using the Mann-Whitney $\mathrm{U}$ test. A value of $p<0.05$ was considered statistically significant.

\section{Results}

A total of 56 newborns were included, 29 in the ultrasound group and 27 in the control group. Figure 2 shows the flow chart for patients. No patient was lost due to death or dropout during the study. Descriptive population data are included in Table 1. There were no differences between both groups as far as the main clinical characteristics, including gender, weight, GA and Apgar score. Figure 3 shows the LUS images of a patient who received surfactant and another who did not.

Surfactant therapy was required in $41.3 \%$ of patients in the ultrasound group and in $62.9 \%$ of patients in the control group $(p=0.106)$. Surfactant administration occurred earlier in the ultrasound group than in the control group, with statistically significant differences: $1 \mathrm{~h}$ of life (IQR 1-2) vs. $6 \mathrm{~h}$ (IQR 4 6.5 ), with $p<0.001$. Figure 4 represents the proportion of patients who received early surfactant therapy (in the first $3 \mathrm{~h}$ of life) in each group. All patients in the ultrasound group received surfactant therapy based on the LUS score threshold.

Table 2 summarises the results of the secondary outcomes. Patients in the ultrasound group had better oxygenation after surfactant therapy, with lower $\mathrm{FiO}_{2}$ requirements and a better $\mathrm{S} / \mathrm{F}$. However, no differences were detected regarding respiratory support (days of non-invasive and invasive mechanical ventilation, ventilator-free days and duration of oxygen therapy) or frequency of bronchopulmonary dysplasia.

The frequency of complications after surfactant administration was no different between groups: desaturation occurred in $4(30.8 \%)$ patients in the ultrasound group and in $6(37.5 \%)$ in the control group, bradycardia in $1(7.7 \%)$ patient in the ultrasound group and in $2(12.5 \%)$ in the control group and apnoea in $1(6.3 \%)$ newborn in the control group (all $p=1.000$ ). The median duration of the LUS scan was 2 min (IQR 2-3).

\section{Discussion}

This study demonstrates that the use of LUS improves the timeliness of surfactant administration without increasing the number of patients requiring surfactant treatment. To our knowledge, this is the first study to assess LUS score thresholds vs. $\mathrm{FiO}_{2}$ requirement thresholds only to guide surfactant replacement therapy in a clinical trial.

Moreover, patients receiving surfactant in the ultrasound group had lower $\mathrm{FiO}_{2}$ requirements, better oxygenation $(\mathrm{S} / \mathrm{F}$ ratio) and better blood gases when surfactant was administered. No patient in the ultrasound group received surfactant as determined by the $\mathrm{FiO}_{2}$ requirement thresholds.

LUS is a simple bedside tool that is easy to perform and is quick (our results show that the procedure lasts $2 \mathrm{~min}$ ), has a high interobserver agreement among clinicians [23] irrespective of observer expertise and can be repeated several times. On an LUS, patients with RDS show an irregular, thickened pleural line, an absence of A lines and coalescent B lines showing [24] white lung. This pattern's severity is scored according to the areas involved [24-26]. Moreover, different studies in premature newborns have shown its correlation with oxygenation $[13,14]$ and with the quality of the endogenous 
Fig. 2 Flow chart of patients

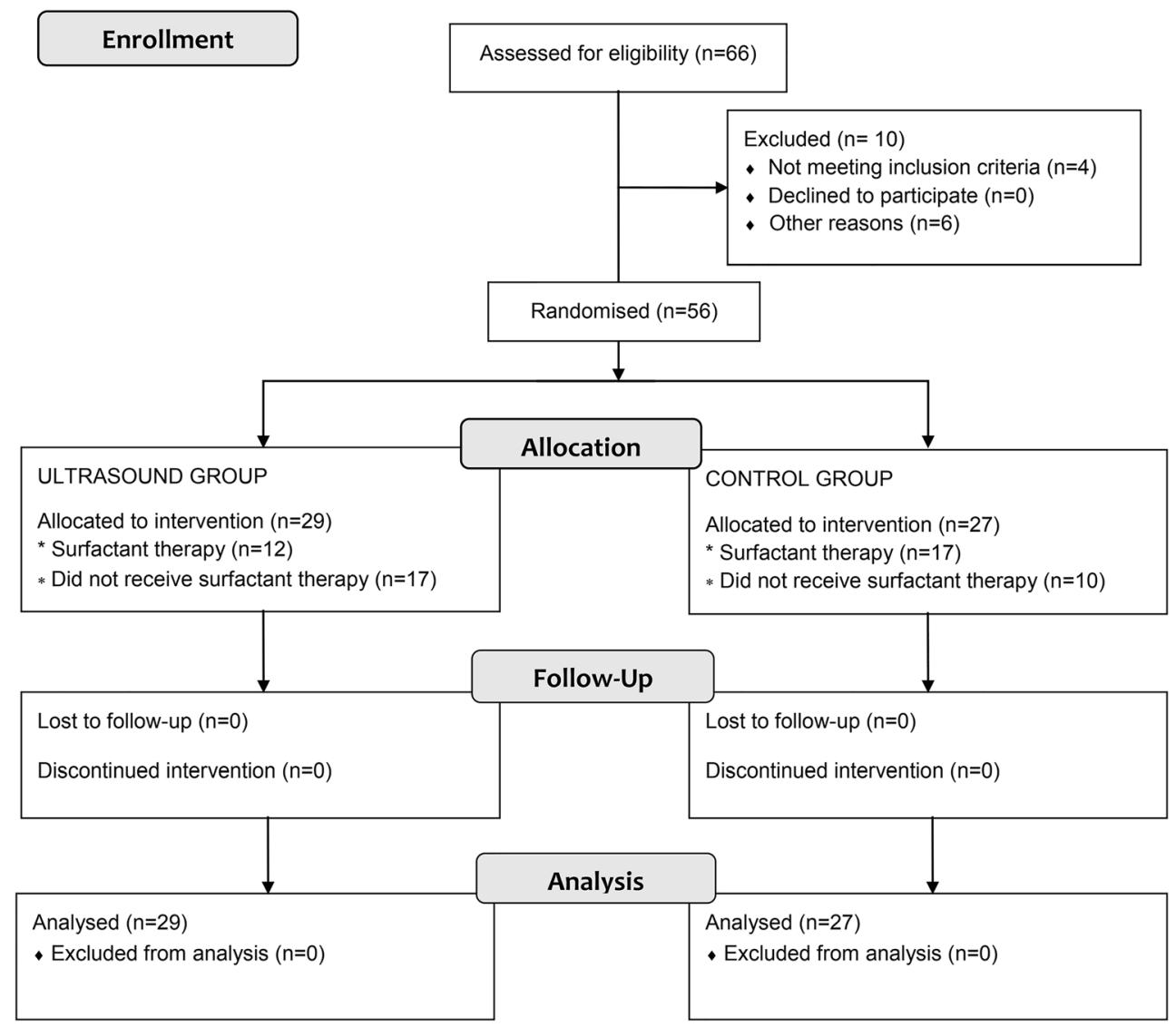

surfactant available [27]. In addition, LUS score has been validated as being on par with other imaging techniques such as CT scans in adults [28].

Our findings are consistent with previous studies published on this matter. In their study, Raschetti et al. [16] found an improvement in early surfactant administration without encountering problems when patients were treated according to LUS instead of $\mathrm{FiO}_{2}$. This data is confirmed by our results: patients in the ultrasound group were in better oxygenation conditions after surfactant treatment, receiving lower oxygen
Table 1 Comparison of both groups regarding clinical data prior to surfactant therapy, including oxygenation parameters

\begin{tabular}{llll}
\hline & Ultrasound group $(n=29)$ & Control group $(n=27)$ & $p$ \\
\hline Male & $16(55.2 \%)$ & $15(55.6 \%)$ & 0.977 \\
Weight $(\mathrm{g})$ & $1500(1058-1808)$ & $1520(1245-1795)$ & 0.909 \\
$\mathrm{GA}$ (weeks) & $30^{1}\left(28^{4}-31^{3}\right)$ & $30^{2}\left(28^{2}-31^{4}\right)$ & 0.669 \\
Intrauterine growth restriction & $3(10.3 \%)$ & $3(11.1 \%)$ & 1.000 \\
Prenatal steroids (complete dose) & $27(93.1 \%)$ & $24(88.9 \%)$ & 0.664 \\
Caesarean section & $13(44.8 \%)$ & $12(44.4 \%)$ & 1.000 \\
APGAR-5 score (points) & $9(8-10)$ & $9(8-10)$ & 0.609 \\
Paw before surfactant $\left(\mathrm{cmH}{ }_{2} \mathrm{O}\right)$ & $6(6-6)$ & $6(6-6)$ & 0.270 \\
$\mathrm{CO}_{2}$ before surfactant $(\mathrm{mmHg})$ & $48(46-51.5)$ & $54(49-58)$ & 0.011 \\
$\mathrm{pH}_{\text {before surfactant }}$ & $7.29(7.27-7.3)$ & $7.26(7.25-7.29)$ & 0.056 \\
$\mathrm{Maximum}$ FiO $_{2}$ in the first hour of life $(\%)$ & $25(22.5-25)$ & $30(25-35)$ & 0.016 \\
$\mathrm{SpO}_{2}$ in the first hour of life $(\%)$ & $94(92.5-95)$ & $93(90-96)$ & 0.572 \\
$\mathrm{~S} / \mathrm{F}$ before surfactant & $380(372-411.5)$ & $340(300-423)$ & 0.108 \\
\hline
\end{tabular}

Data are expressed as mean (standard deviation) or median $\left(25^{\text {th }}-75^{\text {th }}\right.$ percentile) and number $(\%)$ as appropriate. GA gestational age, Paw mean airway pressure, $\mathrm{S} / \mathrm{F} \mathrm{SpO}_{2} / \mathrm{FiO}_{2}$ ratio. $\mathrm{CO}_{2}$ and $\mathrm{pH}$ were analysed using arterialised capillary blood samples 
Fig. 3 a Lung ultrasound of a newborn with an LUS score $<8$. $\mathbf{b}$ Lung ultrasound of a newborn with an LUS score $>8$
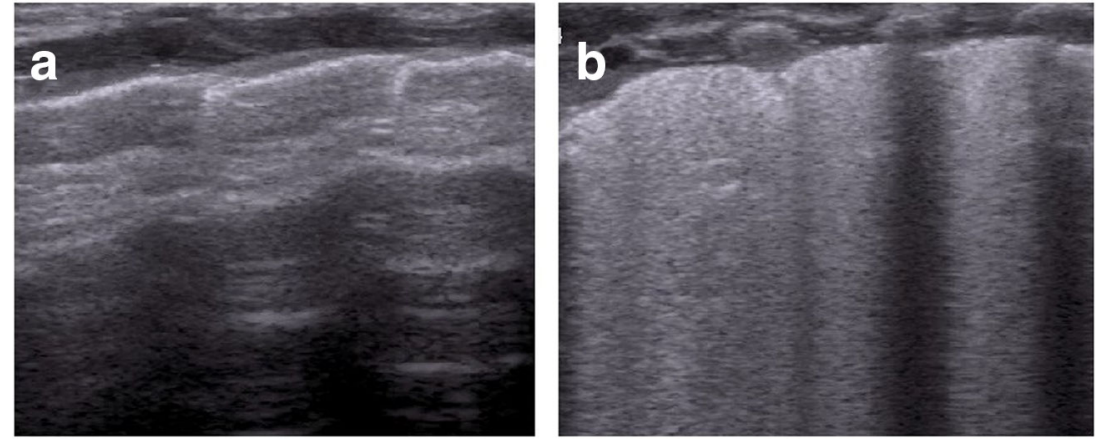

therapy ( $25 \%$ vs. $30 \%$ ) and having a better $\mathrm{S} / \mathrm{F}$ ratio. $\mathrm{CO}_{2}$ was higher in the control group, indicating a more severe RDS and that surfactant replacement should have been administered earlier in this group. The diffusion of $\mathrm{CO}_{2}$ is 25 times higher than $\mathrm{O}_{2}$ diffusion in restrictive disorders, so this is consistent with the higher $\mathrm{FiO}_{2}$ requirements observed in the control group. The lower $\mathrm{S} / \mathrm{F}$ ratio observed after surfactant therapy in both groups could be related to the number of patients who presented desaturation after the procedure, since oxygenation was measured very close to the surfactant administration and no serial evaluation was performed. Despite the better oxygenation parameters, we did not find any improvement in respiratory support, such as later events (sepsis, extrapulmonary disorders). This may be explained by the small sample size of our study and by several other variables that may affect the need for respiratory support. Moreover, it was found that patients in the ultrasound group received surfactant earlier and the maximal $\mathrm{FiO}_{2}$ requirement reached was lower ( $28 \%$ vs. $35 \%)$, without encountering any differences in the overall need for surfactant. The use of LUS score in the ultrasound group resulted in earlier surfactant administration. The timeliest surfactant administration may be achieved by using LUS, not just using $\mathrm{FiO}_{2}$ by itself as has been done up to now. The low rate of timely surfactant administration in the control group in comparison with other studies [16] may be due to our CXR procedure: in most cases, the surfactant treatment was not initiated until the CXR was taken and evaluated. Therefore, we believe that LUS can be extremely useful for all NICUs that tend to have a delayed CXR time.

It is well known that oxygen treatment in premature newborns leads to oxidative stress, damaging proteins, lipids and DNA and placing these fragile preterm patients at high risk for epigenetic changes to the DNA $[18,29]$. As there is not yet a well-established oxygen saturation target and the cut-off value for surfactant treatment may be arbitrary, these patients may receive higher oxygen content. This can trigger the activation of pro-inflammatory and pro-apoptotic pathways, which may contribute to bronchopulmonary dysplasia and other pathologies [30] before surfactant treatment is given during the first hours of life. By contrast, basing the surfactant treatment on LUS score seems to decrease the maximal $\mathrm{FiO}_{2}$ and the duration of oxygen treatment and may help to decrease oxygen toxicity.

Multiple studies have evaluated the use of LUS to predict the need for surfactant or non-invasive ventilation failure in patients with RDS [10-12]. Moreover, LUS seems to predict the need for surfactant administration better than $\mathrm{FiO}_{2}$. Also, LUS may help to identify those patients who do not require surfactant
Fig. 4 Pie graph representing the proportion of patients who received early surfactant therapy (within the first $3 \mathrm{~h}$ of life) in each group

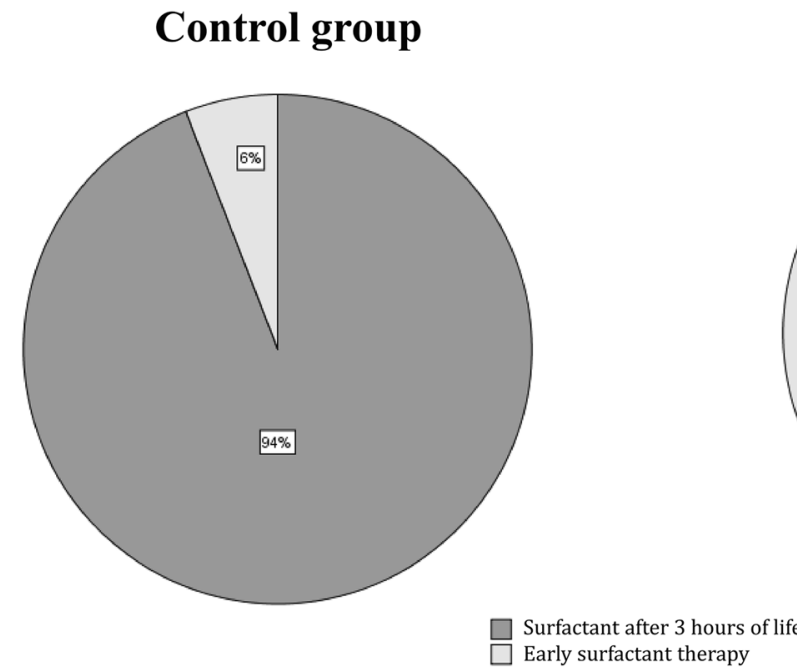

Ultrasound group

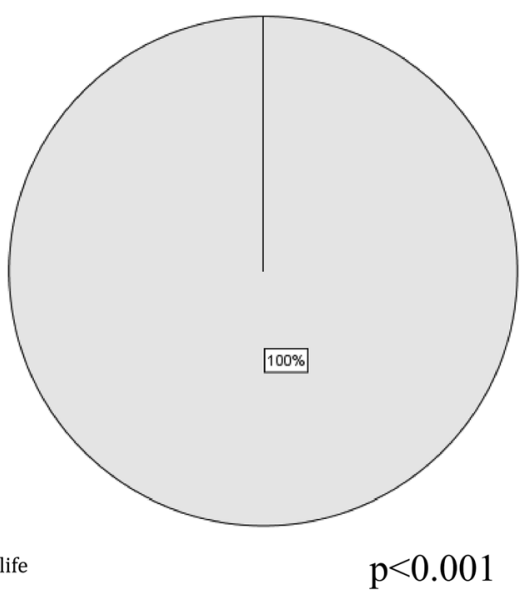


Table 2 Summary of the secondary outcomes, including the respiratory requirements and oxygen exposure in early life

\begin{tabular}{llll}
\hline & $\begin{array}{l}\text { Ultrasound group } \\
(n=29)\end{array}$ & $\begin{array}{l}\text { Control group } \\
(n=27)\end{array}$ & $p$ \\
\hline $\mathrm{FiO}_{2}$ after surfactant (\%) & $28(25-30)$ & $35(31-40)$ & $<0.001$ \\
$\mathrm{SpO}_{2}$ after surfactant (\%) & $93.5(92-95)$ & $90(90-90.8)$ & 0.001 \\
$\mathrm{~S} / \mathrm{F}$ after surfactant & $345(290-381)$ & $285(241-300)$ & 0.012 \\
$\mathrm{MV}$ & $5(17.2 \%)$ & $10(37.0 \%)$ & 0.095 \\
Duration of MV (days) & $2(1-4.5)$ & $2(1.8-4.3)$ & 0.523 \\
Ventilator-free days & $28(28-28)$ & $28(26-28)$ & 0.082 \\
NIV & $29(100 \%)$ & $27(100 \%)$ & 1.000 \\
Duration of NIV (days) & $3(2-17)$ & $4(3-22)$ & 0.428 \\
Duration of oxygen (days) & $6(2-23.5)$ & $5.5(3-22.8)$ & 0.926 \\
Length of stay in the NICU (days) & $47(34-69)$ & $52(38-68)$ & 0.780 \\
BPD & $3(10.3 \%)$ & $3(11.1 \%)$ & 1.000 \\
\hline
\end{tabular}

Data are expressed as mean (standard deviation) or median $\left(25^{\text {th }}-75^{\text {th }}\right.$ percentile) and number $(\%)$ as appropriate. $B P D$ bronchopulmonary dysplasia, $M V$ invasive mechanical ventilation, $N I C U$ neonatal intensive care unit, $N I V$ non-invasive mechanical ventilation, and $S / F \mathrm{SpO}_{2} / \mathrm{FiO}_{2}$ ratio treatment and may help clinicians to identify other causes, apart from RDS, of hypoxia and non-invasive ventilation failure, so they can treat the patient accordingly [23, 31, 32].

Due to our study protocol, patients intubated in the delivery room or transferred from other centres were excluded; this may explain the early surfactant administration in both groups (median $1 \mathrm{~h}$ vs. $6 \mathrm{~h}$ ) when compared with other studies $[11,14]$.

Our study has several limitations. Firstly, it was carried out at a single centre and our sample size is small. In addition, not all the patients received surfactant treatment. However, differences in the need for surfactant administration between both groups were not statistically significant, and earlier treatment was demonstrated in the ultrasound group. Another limitation is that although we included premature newborns younger than 32 weeks, including extremely premature ones (GA < 28 weeks), it would have been better to classify the patients according to their GA, but this would have required a larger, randomised multicentre study. Another limitation was that the attending clinicians were not blinded to the ultrasound findings. However, we enrolled a homogeneous population of preterm newborns with a GA between 24 and 32 weeks with RDS who were all treated according to the same NICU protocol, with LUS performed at a specific time in the first hour, thus decreasing the possible variances in the LUS score. In addition, we did not find any differences in the overall need for surfactant in both groups, which rules out the possibility of surfactant being given to patients who did not require it. Since LUS is routinely used in our unit as the first-line chest imaging technique and the medical team has considerable knowledge regarding ultrasounds, our results may be different from those obtained in other units where LUS is less frequently used (or may be more difficult to apply) and where CXR remains the imaging technique of choice.
Therefore, it has been shown that LUS improves timeliness in surfactant replacement. Larger, randomised multicentre studies are likely necessary to evaluate its use more in depth, especially in very premature patients under 28 weeks. Our results may help to continue the investigation into the use of LUS to guide surfactant therapy in conjunction with $\mathrm{FiO}_{2}$ parameters.

Acknowledgements We would like to thank the premature newborns and families who accepted joining this study.

Authors' contributions JRF, SBP, ABM and MR conceived and designed the study. MB, IJ and SBP analysed the data. JRF, SBP, MB and IJ wrote the first draft of the manuscript. ABM and MR contributed to the writing of the manuscript. All authors agree with the manuscript's results and conclusions, jointly developed the structure and arguments for the paper, made critical revisions and reviewed and approved the final version of the manuscript.

Funding information There is no external funding source.

\section{Compliance with ethical standards}

Conflict of interest The authors declare that they have no conflicts of interest or financial relationship with any organization.

Ethical approval This study was approved by the local Healthcare Ethics Committee and the institutional review board.

Informed consent Written informed consent was obtained from all the parents of the participants.

\section{References}

1. Aldana-Aguirre C, Pinto M, Featherstone RM, Kumar M (2017) Less invasive surfactant administration versus intubation for 
surfactant delivery in preterm infants with respiratory distress syndrome: a systematic review and meta-analysis. Arch Dis Child Fetal Neonatal Ed 102(2):17-23

2. Sweet DG, Carnielli V, Greisen G, Hallamn M, Ozek E, Te Pas A et al (2019) European consensus guidelines on the management of respiratory distress syndrome-2019 update. Neonatology 115:432451

3. Bahadue FL, Soll R, Cochrane Neonatal group (2012) Early versus delayed selective surfactant treatment for neonatal respiratory distress syndrome. Cochrane Database Syst Rev 11(11):CD001456. https://doi.org/10.1002/14651858

4. Raschetti R, Centorrino R, Letamendia E, Benachi A, MarfaingKoka A, De Luca D (2019) Estimation of early endogenous surfactant pool and CPAP failure in preterm neonates with RDS. Respir Res 20(1):75

5. Gortner L, Schüller SS, Herting E (2018) Review demonstrates that less invasive surfactant administration in preterm neonates leads to fewer complications. Acta Paediatr 107(5):736-743

6. Shari HMM, Naghibi S, Mahdavi E, Khademi G (2014) Diagnostic utility of chest X-rays in neonatal respiratory distress: determining the sensitivity and specificity. Int J Pediatr 2(3):65-72

7. Roggini M, Pepino D, D’Avanzo M, Andreoli GM, Ceccanti S, Capocaccia P (2010) Respiratory distress in newborn: evaluation of chest X-rays. Minerva Pediatr 62:217-219

8. Okterm A, Yigit S, Oguz B, Celik T, Halilogu M, Yurdakok M (2019) Accuracy of lung ultrasonography in the diagnosis of respiratory distress syndrome in newborns. J Matern Fetal Neonatal Med 22:1-6

9. Liu Lung ultrasonography for the diagnosis of neonatal lung disease. J Matern Neonatal Med 27(8):856-861

10. Rodriguez-Fanjul J, Balcells C, Aldecoa-Bilbao V, Moreno J, Iriondo M (2016) Lung ultrasound as a predictor of mechanical ventilation in neonates older than 32 weeks. Neonatology 110(3): 198-203

11. Gregorio-Hernández R, Arriaga-Redondo M, Pérez-Pérez A, Ramons-Navarro C, Sánchez-Luna M (2020) Lung ultrasound in preterm infants with respiratory distress: experience in a neonatal intensive care unit. Eur J Pediatr 179(1):81-89

12. Corsini I, Parri N, Gozzini E, Coviello C, Leonardi V, Poggi C, Giacalone M, Bianconi T, Tofani L, Raimondi F, Dani C (2019) Lung ultrasound for the differential diagnosis of respiratory distress in neonates. Neonatology 115(1):77-84

13. DeMartino L, Yousef N, Ben-Ammar R, Raimondi F, ShankarAguilera S, De Luca D (2018) Lung ultrasound score predicts surfactant need in extremely preterm neonates. Pediatrics 142(3)

14. Brat R, Yousef N, Klifa R, Reynaud S, Shankar Aguilera S, De Luca D Lung ultrasonography score to evaluate oxygenation and surfactant need in neonates treated with continuous positive airway pressure. JAMA Pediatr 169(8):e151797. https://doi.org/10.1001/ jamapediatrics.2015.1797

15. Razak A, Faden M (2020) Neonatal lung ultrasonography to evaluate need for surfactant or mechanical ventilation: a systematic review and meta-analysis. Arch Dis Child Fetal Neonatal Ed 105(2):164-171

16. Raschetti R, Yousef N, Vigo G, Marseglia G, Centorrino R, BenAmmar R (2019) Echography-guided surfactant therapy to improve timeliness of surfactant replacement: a quality improvement project. J Pediatr 212:137-143

17. Tipple TE, Ambalayanan N (2019) Oxygen toxicity in the neonate: thinking beyond the balance. Clin Perinatol 46(3):435-447

18. De Luca D, van Kaam AH, Tingay DG, Courtney SE, Danhaive O, Carnielli VP et al (2017) The Montreux definition of neonatal
ARDS: biological and clinical background behind the description of a new entity. Lancet Respir Med 5(8):657-666

19. Dell'Orto V, Raschetti R, Centorrino R, Montane A, Tissieres P, Yousef N, Luca D (2019) Short and long-term respiratory outcomes in neonates with ventilator-associated pneumonia. Pediatr Pulmonol 54(12):1982-1988

20. Higgins RD, Jobe AH, Koso-Thomas M, Bancalari E, Viscardi RM, Hartert TV, Ryan RM, Kallapur SG, Steinhorn RH, Konduri GG, Davis SD, Thebaud B, Clyman RI, Collaco JM, Martin CR, Woods JC, Finer NN, Raju TNK (2018) Bronchopulmonary dysplasia: executive summary of a workshop. J Pediatr 197:300-308

21. Khemani RG, Smith LS, Zimmerman JJ, Erickson S, Pediatric Acute Lung Injury Consensus Conference Group (2015) Pediatric acute respiratory distress syndrome: definition, incidence, and epidemiology: proceedings from the Pediatric Acute Lung Injury Consensus Conference. Pediatr Crit Care Med 16:23-40

22. Cogo PE, Facco M, Simonato M, De Luca D, De Terlizi F, Rizzotti U et al (2011) Pharmacokinetics and clinical predictors of surfactant redosing in respiratory distress syndrome. Intensive Care Med 37(3):510.517

23. Gomond-Le Goff C, Vivalda L, Foligno S, Loi B, Yousef N, De Luca D Effect of different probes and expertise on the interpretation reliability of point-of-care lung ultrasound. Chest. https://doi.org/ 10.1016/j.chest.2019

24. Bobillo-Perez S, Girona-Alarcon M, Rodriguez-Fanjul J, Jordan I, Balaguer GM (2019) Lung ultrasound in children: what does it give us? Paediatr Respir Rev. https://doi.org/10.1016/j.prrv.2019.09.006

25. Vergine M, Copetti R, Brusa G, Cattarossi L (2014) Lung ultrasound accuracy in respiratory distress syndrome and transient tachypnea of the newborn. Neonatology 106(2):87-93

26. Raimondi F, Migliaro F, Verdoliva L, Gragnaniello D, Poggi G, Kosova R, Sansone C, Vallone G, Capasso L (2018) Visual assessment versus computer-assisted gray scale analysis in the ultrasound evaluation of neonatal respiratory status. PLoS One 13(10): e0202397

27. Autilio C, Echaide M, Benachi A, Marfaing-Koka A, Capoluongo ED, Pérez-Gil J, de Luca D (2017) A noninvasive surfactant adsorption test predicting the need for surfactant therapy in preterm infants treated with continuous positive airway pressure. J Pediatr 182:66-73

28. Chiumello D, Mongodi S, Algieri I, Vergani GL, Orlando A, Via G, Crimella F, Cressoni M, Mojoli F (2018) Assessment of lung aeration and recruitment by CT scan ultrasound in acute respiratory distress syndrome patients. Crit Care Med 46:1761-1768

29. Torres-Cuevas I, Parra-Llorca A, Sánchez-Illana A, Nuñez-Ramiro A, Kuligowski J, Cháfer-Pericás C, Cernada M, Escobar J, Vento M (2017) Oxygen and oxidative stress in the perinatal period. Redox Biol 12:674-681

30. Perrone S, Laschi E, Buonocore G (2019) Biomarkers of oxidative stress in the fetus and in the newborn. Free Radic Biol Med 142:2331

31. Bookman L, Troy R, McCaffrey M, Randolph G (2010) Using quality improvement methods to reduce variation in surfactant administration. BMJ Qual Saf 19:e23

32. Tusor N, De Cunto A, Basma Y, Klein J, Meau-Petit V (2020) Ventilator-associated pneumonia in neonates: the role of point of care lung ultrasound. Eur J Pediatr 26:1-10

Publisher's note Springer Nature remains neutral with regard to jurisdictional claims in published maps and institutional affiliations. 